\title{
Dependence of mass in comparison to full width of mesons
}

\author{
Anatoliy Nedolya ${ }^{1 \star}$
}

${ }^{1}$ Zaporizhzhya National University, 66 Zhukovsky str., 69600 Zaporizhzhya, Ukraine

\begin{abstract}
The mass of mesons in comparison to their full width (lifetime) using the Review of Particle Physics (Particle Data Group) was phenomenologically investigated. The dependence showed that most of mesons were located inside the range, the upper edge of which varied periodically and looked like waves' superposition. Similar distribution was observed for baryons. Such uneven distribution of mesons and baryons explains discrete distribution of particles' mass and energy in the Universe. The results may be useful to clarify the range of mass for searching new particles.
\end{abstract}

\section{Introduction}

It is known that the lifetime of relativistic particles depends on their energy. For example, the lifetime of muons in cosmic rays increases by Lorentz factor $\gamma=\left(1-\beta^{2}\right)^{-1 / 2}$ times. Based on uncertainty principle, for the short-lived particles the lifetime is determined from the full width $(\Gamma=\hbar / \tau)$ of energy distribution of the decaying particles. However, there is a problem of formal description of the unstable states using quantum mechanics and quantum field theory, because such description with the finite lifetime of unstable particle contradicts the notion of asymptotic state [1]. Currently the dependence of elementary particle's lifetime on its mass (rest energy) is not determined, although the particles' lifetime depends on the type of their fundamental interaction. Actually the particles that decay due to strong interaction normally do so in less than $10^{-20} \mathrm{~s}$; the decay caused by electromagnetic interaction generally have lifetimes in the order of $10^{-16} \mathrm{~s}$, the weak interaction decay takes longer than $10^{-10} \mathrm{~s}$ $[2,3]$.

\section{Results}

Description of unstable particles by methods of quantum field theory is associated with the value of $\Gamma / M$, which for stable and quasi-stable particles is very small, but it has essential value for most of hadrons. That is why in this study the full width (lifetime) of mesons in comparison to their masses was investigated using the last Particle Data Group [4].

Figure 1 shows that most of mesons were located inside range, upper edge of which varied periodically and the dependence was similar to a result of waves' superposition. The values of masses with a maximum of full widths relate to each other as $1,2,3$, and possibly 6 with a period near to $2 \mathrm{GeV}$. Here were denoted: 1 - unflovered + strange maximum; 2 - $c \bar{c}$ maximum (charmonium); 3 -

^e-mail: avnedolya@hotmail.com 


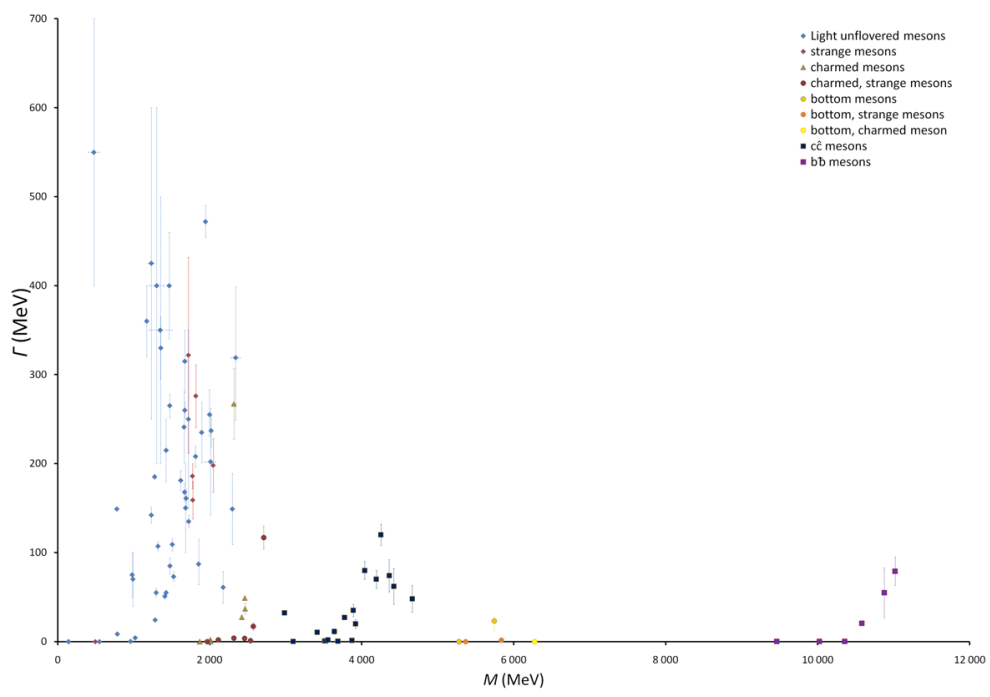

Figure 1: Masses of mesons in comparison to their full widths.

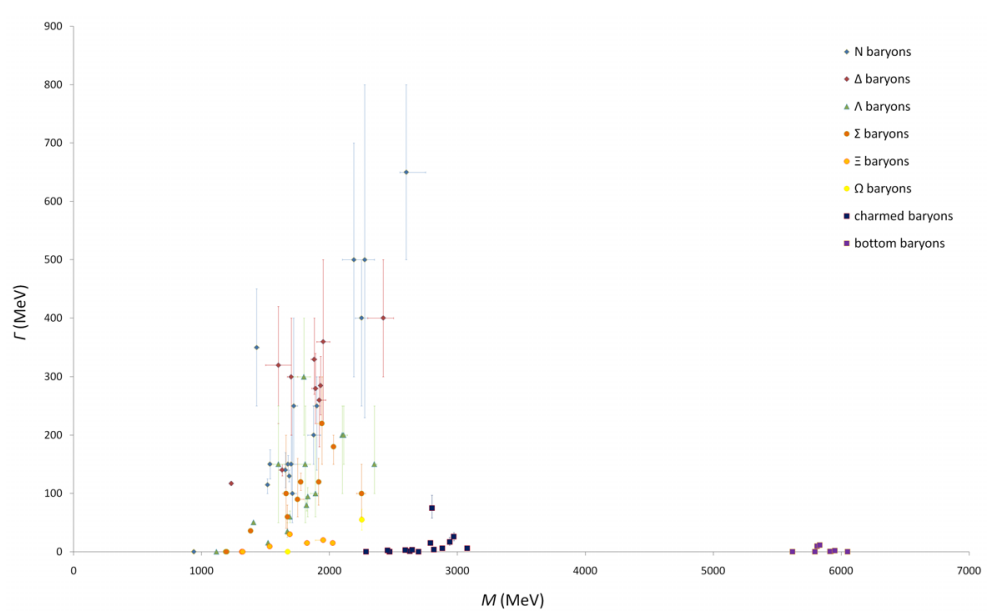

Figure 2: Masses of baryons in comparison to their full widths.

bottom maximum; $6-b \bar{b}$ possible maximum (bottonium). No mesons have been found in the mass range from 6300 to $9400 \mathrm{MeV}$. Only $f_{0}(500)$ unflavored meson was located outside that range and its error values were big for mass and full width. Their data should be revised. Similar distribution was observed for baryons (see Fig. 2).

Figure 2 includes the data of Breit-Wigner mass and Breit-Wigner full width for $N$ and $\Delta$ baryons, but their description may be only approximate and may contain some contradiction since PDG lists in addition another value, which is called pole position [5]. But the overal $\Gamma(M)$ dependence for baryons is periodical and also resembles waves' superposition, where the mass and full width values 


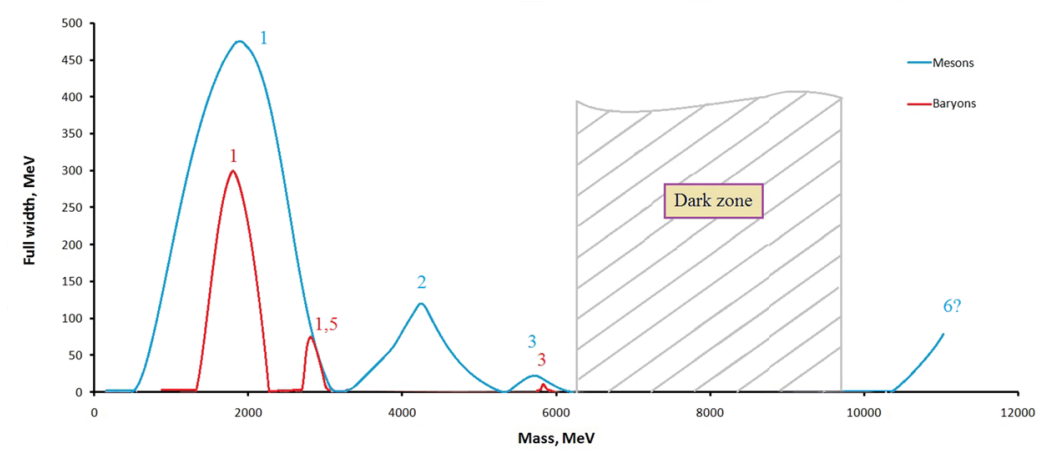

Figure 3: $\Gamma(M)$ schematic result of waves' superposition for hardrons.

are related to each other as 1, 1.5, 3 and where 1 is a baryons maximum; 1.5 - charmed baryons, 3 bottom baryons maximum respectively.

Taking into account these data a tentative scheme of $\Gamma(M)$ dependence (see Fig. 3) was offered, which illustrated the general distribution of hadrons masses vs. their full widths. As a result the maximums are not always matched to flavor of the resonances and also may include the tetra- and pentaquark moleculas data $[6,7]$. There is a wide range of unobserved values of mass (absorption band of spectrum), which is described as the Dark zone.

\section{Summary}

Thus it is possible that the hadrons are decaying with most probability at some values of mass, which are coinciding with the superposition's maximums of $\Gamma(M)$ dependence. It is a result of Higgs field interaction. It shows that most likely, mass and energy are distributed unevenly in the Universe. In addition, the $\Gamma(M)$ dependence can also explain different interaction radius of elementary particles which have a mass. Thus, conceptually the Higgs boson, which gives mass to elementary particles, may be more universal. The existence of a dark zone remains an open question and its can be regarded as absorption band of masses or range in which the particles may decay so quickly that modern equipment can not detected them. The results could be useful to clarify the mass range for searching new particles.

\section{References}

[1] V.I. Kuksa, Phys. Part. Nuclei 45 (3), 568 (2014)

[2] D. Griffiths, Introduction to Elementary Particles (Wiley-VCH GmbH \& Co. KGaA, Weinheim, 2004) 72

[3] S.T. Thornton, A. Rex, Modern Physics for Scientists and Engineers, Forth Edition (Books/Cole, Boston, 2013) 530

[4] K.A. Olive et al. Chinese Physics C 38 (9), 090001 (2014)

[5] A.R. Bohm, Y. Sato, Phys. Rev. D 71, (2005) 085018

[6] G. Cowan (2016) CERN see https://cds.cern.ch/record/2156233?ln=en

[7] H.X. Chen et al. Physics Report 639, 1 (2016) 